\title{
Insertion depths of RAE tracheal tubes for orotracheal intubation according to fixation site and head position changes
}

\author{
Kang J.-M., Jung H.-M., Kangdong Kyunghee University Hospital, Dept of Anaesthesiology \& Pain Medicine, \\ Seoul, Korea, Republic of
}

Background and Goal of Study: The use of RAE tubes in head and neck surgery has many advantages. The tubes lay along the chin without obstructing the view of the surgical field. We compared the insertion depth of the RAE tube for orotracheal intubation by using flexible fiberoptic bronchoscope when fixed at the midline and right angle of the mouth in the simple supine position and again following extension of the neck.

Materials and Methods: One hundred adults (50 males, 50 females) presenting for routine elective surgery under general anesthesia were studied. Patients were placed on the operating table with the head in the neutral position. Routine monitors were used. Induction of anesthesia was achieved with $1.5 \mathrm{mg} / \mathrm{kg}$ propofol IV. Muscle relaxation was obtained with $0.6 \mathrm{mg} / \mathrm{kg}$ rocuronium IV. Anesthesia was maintained with sevoflurane (2-3\%) and remifentanil (0.1-0.2 $\mu \mathrm{g} / \mathrm{kg} / \mathrm{min})$. After los of all four twitches on the train-of-four obtained by ulnar nerve stimulation, male patients were intubated with a 7.5 mm inner diameter (ID) RAE tube (RAE tube, Mallinckrodt Medical, Tullamore, Ireland), while $7.0 \mathrm{~mm}$ ID RAE tubes were used for female patients. One small black band is printed on the product itself at $22 \mathrm{~cm}$ from the distal end of the tube for a $7.5 \mathrm{ID}$ RAE tube and $21 \mathrm{~cm}$ for a $7.0 \mathrm{ID}$ RAE tube. Fixation of RAE tubes was performed just at this black band on the right angle of the tube. A fiberoptic bronchoscope was introduced through the swivel connector into the RAE tube and the distances from the swivel connector to the landmark (incisor or canine), tip of tube and the carina were measured with the RAE tube in the midline and at the right angle of the mouth. An assistant helped the black band on the tube stay firmly at the landmark during measurement. These procedures were also performed in Rose's position (head hyperextension position) with a small pillow under the patient's chest.

The distance from the upper incisor to the tracheal tube tip (incisor-tube tip length) was calculated from the difference between the distances from the swivel connector to the upper incisor and the tracheal tube tip. The distance from the upper incisor to carina (incisor-carina length) was calculated from the difference between the distances from the swivel connector to the upper incisor and the carina. The distance from the tube tip to the carina (tube tip-carina length) could be calculated as the difference between the above two distances.

Results and Discussion: In males, the tip to carina distances increased from $4.5 \mathrm{~cm}$ to $6.4 \mathrm{~cm}$ in the neutral head position, and from $5.8 \mathrm{~cm}$ to $7.4 \mathrm{~cm}$ in the extended head position with moving the fixation site from right canine to upper incisor. In females, the tip to carina distances increased from $3.4 \mathrm{~cm}$ to $4.6 \mathrm{~cm}$ and from $4.9 \mathrm{~cm}$ to $6.2 \mathrm{~cm}$ in each position respectively with the same action $(\mathrm{P}<0.001)$.

The RAE orotracheal tube was specifically designed for use with the Boyle-Davis mouth gag holding the mouth open during oral surgery. The RAE tube needs to be secured at the midline or right mouth angle and to be applied to various head positions depending on the type of surgery. We have a tendency to fix the RAE tube at the preformed bend in an unconcerned manner without considering the specific condition of the intubation or surgery. Moreover it is somewhat uncomfortable to fix the RAE tube at any spots other than the bending point of the tube whether it is proximal or distal to the bending point. There are few studies about the insertion depth of RAE tube regarding the fixation site or head position although it has been widely used. This study was performed to find out the difference in depth according to tube fixation site or position change.

A standard point for the fixation of the tubes can be lip or teeth at the right angle or the midline of the mouth. We chose the upper incisor and the right canine as the fixation landmarks in the current study because lip could be redundant and movable while measuring the distances with a fiberoptic brochoscope.

Many ENT surgeries including tonsillectomy require Rose's position to visualize the orophaynx or laryngeal structures. Our study showed that in males, the tip to carina distances of the RAE tubes increased by $1.9 \mathrm{~cm}$ in the neutral head position and $1.6 \mathrm{~cm}$ in the hyperextended head position with moving the fixation site from right canine to upper incisor. In females, the tip to carina distances increased by $1.2 \mathrm{~cm}$ in the neutral head position and $1.3 \mathrm{~cm}$ in the hyperextended head position with the same movement of the tubes. In addition, it was also reconfirmed to be true that hyperextension of head itself increases the tip to carina distance with the tube fixed at the same landmark.

The tube tip is recommended to be 4 or $5 \pm 2 \mathrm{~cm}$ from carina to avoid being inserted too shallow or far. Previous studies showed us that it is safe and rational to determine the insertion depth of the plain endotracheal tube on a personal basis because airway lengths are different among individuals. If the RAE tube is fixed at its own pre-bended points to all the patients in undifferentiated fashion, it will be too short or deep in some patients. This can be worsened depending on the fixation site (midline or mouth angle) or position of the patient (neutral or extension). The current results would be helpful to modifying the tube insertion depth needed for these changes.

Conclusion(s): There was a significant increase in the tip to carina distance of the RAE tubes with changing the fixation site of endotracheal tube from right canine to upper incisor in both neutral and extended head positions of the adults.

References:

1. Cherng CH, Wong CS, Hsu CH, Ho ST. Airway length in adults: estimation of the optimal endotracheal tube length for orotracheal intubation. J Clin Anesth 2002 14: $271-4$.

2. Sharma K, Varshney M, Kumar R. Tracheal tube fixation: the effect on depth of insertion of midline fixation compared to the angle of the mouth. Anaesthesia 2009; 64: 383-6.

3. Conrardy PA, Goodman LR, Lainge F, Singer MM. Alteration of endotracheal tube position. Flexion and extension of the neck. Crit Care Med 1976; 4: 7-12.

\begin{tabular}{|c|c|c|}
\hline & Men $(\mathrm{n}=50)$ & Women $(\mathrm{n}=50)$ \\
\hline Age $(\mathrm{yr})$ & $40 \pm 17$ & $44 \pm 17$ \\
\hline Height $(\mathrm{cm})$ & $171 \pm 6$ & $159 \pm 6$ \\
\hline Weight $(\mathrm{kg})$ & $72 \pm 9$ & $58 \pm 9$ \\
\hline
\end{tabular}

Table 1. Patient Characteristics. Values are mean \pm SD.

\begin{tabular}{|c|c|c|c|c|c|c|c|c|c|c|c|c|}
\hline \multirow{3}{*}{ Distances } & \multicolumn{6}{|c|}{ Men $(n=50)$} & \multicolumn{6}{|c|}{ Women $(\mathrm{n}=50)$} \\
\hline & \multicolumn{3}{|c|}{ Neutral } & \multicolumn{3}{|c|}{ Extended } & \multicolumn{3}{|c|}{ Neutral } & \multicolumn{3}{|c|}{ Extended } \\
\hline & angle & midline & $\mathrm{P}$ & angle & midline & $\mathrm{P}$ & angle & midline & $\mathrm{P}$ & angle & midline & $\mathrm{P}$ \\
\hline Teeth to tube tip & $20.9 \pm 0.4$ & $21.0 \pm 0.3$ & & $20.9 \pm 0.4$ & $21.0 \pm 0.3$ & & $19.8 \pm 0.3$ & $19.9 \pm 0.2$ & & $19.9 \pm 0.3$ & $20.0 \pm 0.4$ & \\
\hline Teeth to carina & $25.5 \pm 1.2$ & $27.4 \pm 1.3$ & $<0.001$ & $26.7 \pm 1.5$ & $28.4 \pm 1.5$ & $<0.001$ & $23.2 \pm 1.7$ & $24.5 \pm 1.7$ & $<0.001$ & $24.9 \pm 2.1$ & $26.2 \pm 1.6$ & $<0.001$ \\
\hline Tube tip to carina & $4.5 \pm 1.3$ & $6.4 \pm 1.3$ & $<0.001$ & $5.8 \pm 1.5$ & $7.4 \pm 1.6$ & $<0.001$ & $3.4 \pm 1.7$ & $4.6 \pm 1.7$ & $<0.001$ & $4.9 \pm 2.0$ & $6.2 \pm 1.3$ & $<0.001$ \\
\hline
\end{tabular}

Table 2. Comparison of the airway distances measured with the RAE tube fixed in the midline or at the angle of the mouth in males and females. Values are mean \pm SD. 